\section{Pflegereform im Rahmen des} Möglichen?

Glaubt man den Meinungsforschern, stehen „Gesundheit und Pflege“ bei den Wählern oben an. Bei Demenz im Alter, dämmert es den Menschen, ist Minutenpflege nicht erstrebenswert. Und die Presse illustriert bereits gnadenlos mit Szenen aus dem slowakischen Landheim. Wie könnte Politik da auf einfühlsame Worte verzichten. Und dennoch bleibt die Diskrepanz zwischen empathischer Wähleransprache und sozialem Stillstand in der Pflege mehr als aufreizend. Menschen mit kognitiven und psychischen Beeinträchtigungen tatsächlich bedarfsgerecht in die Pflege einzubeziehen scheut die Politik. Aber auch mit den Mitarbeitern in der Pflege, den sogenannten „Pflegekräften“, findet sie keinen rechten Frieden. Nicht nur, dass von der Wohlfahrtsindustrie selbst magere Tarife nicht eingehalten werden, wegen der Arbeitsbedingungen bleibt auch die geringe Verweildauer im Beruf ein gravierendes Problem.

An Konzepten fehlt es nicht. Zahlreiche Beiräte und Expertenkreise definieren seit Jahren den neuen Pflegebedürftigkeitsbegriff rauf und runter: Sie scheitern schlicht an der Fiskalisierung der Politik. Auf der einen Seite steht trutzig der „Solidarpakt", nach dem die Sozialversicherungsbeiträge in Deutschland möglichst unter 40 Prozent zu halten sind. Auf der anderen Seite baut sich die verfassungsbewehrte Schuldenbremse auf. Dem ohnehin seit Jahren abschmelzenden fiskalischen Spielraum droht ein geradezu inverses Schicksal. In der europäischen Schuldenkrise hat die Politik bisher nur „Zeit gekauft“, ohne allzu viel damit anfangen zu können. $\mathrm{Ab}$ einer Schuldenquote von 90 Prozent des BIP gehe die Fähigkeit zum Wachstum gegen Null, zeigen ökonometrische Modelle. Nicht dass wir uns auf sie verlassen könnten: Aber die Zurückhaltung, mit der in Deutschland zu recht aktuelle Reserven der Sozialhaushalte behandelt werden, spricht Bände. Die Krise ist so komplex, dass Austerität zur Staatsraison wird. Doch kann Austerität als Schimpfwort herhalten, wenn sich die Staaten den internationalen Finanzmärkten bereits restlos ausgeliefert haben?

Es wäre eine Schande für das Land, bliebe für die Pflege wie zuletzt nur die Privatisierung als Ausflucht und die F.D.P. als Hilfsmotor der Sozialreform. Aber der hatte ja am 22. September einen formidablen Kolbenfresser. Andererseits soll doch niemand glauben, die historische Selbstenthauptung der SPD mit HARTZ ${ }^{4}$ und Rente ${ }^{67}$ ließe sich unter dem Kragenspiegel revidieren, wenn ab 2016 die Garrotte der Schuldenbremse greift. Bei weiter abnehmendem fiskalischem Spielraum muss sich die Sozialpolitik vielmehr den Verteilungsfragen neu stellen. So ist etwa die Strategie der Ärzteschaft zu hinterfragen, Einkommenszuwächse durch regelgebundene Forderungen zu immunisieren. Wie das Statistische Bundesamt (StBA) zeigt, haben die Kassenärzte ihren Reinertrag in den letzten 4 Jahren um 17 Prozent gesteigert. Im Schutz der Sozialgesetze - wen sollten die eigentlich schützen? - werden solche Zuwächse mehr und mehr immunisiert, ohne dass die inflationäre Abrechnung überwunden würde oder auch nur die Wartezeiten für die 90 Prozent Kassenpatienten abnähme. Vielmehr betont die Kassenärztliche Bundesvereinigung (KBV) zu den Zahlen des StBA, dass die 90 Prozent Kassenpatienten nur noch mit 68 Prozent zum Umsatz der Praxen beitrügen. Bei den Fachärzten nähert sich die Quote der 50 Prozentmarke. Wegen der reinen Einzelleistungsvergütung bleibt die gesetzliche Krankenversicherung im Wettlauf der Gebührenordnungen von „GKV und PKV“ immer noch zweiter Sieger. Daher fordert die KBV nunmehr, die für Teile der Leistungen noch verbliebenen Budgetdeckel zu entfernen. Die Sicherstellung einer flächendeckenden Versorgung durch Hausärzte und Fachärzte wird dadurch nicht gefördert.

Eine „integrierte Krankenversicherung“, wie sie in dieser Ausgabe skizziert wird, überwindet im Übrigen die morsche Konkurrenz paralleler Gebührenordnungen. Dass damit die gefährdete PKV aufgefangen wird, ist noch der geringste Nutzen. Entscheidend scheint die Chance, zu einer rationalen Gebührenordnung vorzudringen. Sie stellte den Versorgungsbedarf des Patienten als Grundprinzip über die Mechanismen einer institutionell immunisierten Mittelbeschaffung.

Die Herausgeber 